\title{
Tangence
}

\section{Ironie dans la pensée juive et dans la poésie de Benjamin Fondane}

\section{Armelle Chitrit}

Numéro 53, décembre 1996

L’humour de la poésie

URI : https://id.erudit.org/iderudit/025927ar

DOI : https://doi.org/10.7202/025927ar

Aller au sommaire du numéro

Éditeur(s)

Tangence

ISSN

0226-9554 (imprimé)

1710-0305 (numérique)

Découvrir la revue

Citer cet article

Chitrit, A. (1996). Ironie dans la pensée juive et dans la poésie de Benjamin

Fondane. Tangence, (53), 58-75. https://doi.org/10.7202/025927ar d'utilisation que vous pouvez consulter en ligne.

https://apropos.erudit.org/fr/usagers/politique-dutilisation/ 


\section{Ironie dans la pensée juive et dans la poésie de Benjamin Fondane}

\section{Armelle Chitrit}

Benjamin Fondane avait quitté la Roumanie en 1923 dans l'idée de rejoindre Tzara et Dada à Paris :

Car Dada était la première manifestation connue de l'esprit, qui se prétendait catastrophique, qui rejetait toute finalité, qui proclamait le scandale pour le scandale, tout en cachant (ou ignorant peut-être) l'idée obscure qui l'y poussait, qui refusait de se laisser prendre pour un Événement. ${ }^{1}$

Mais Breton et quelques autres, loin de comprendre le projet d'une telle absurdité, exigèrent qu'on traduisît cette "activité spirituelle "sur un plan social et intellectuel. Le surréalisme cherchait des réponses. Mais la trajectoire de Fondane, celle du voyageur comme celle de l'écrivain, restait déterminée à vaincre les effets pervers de la logique, à renverser la dictature de la Raison. Cette mise en doute: pour faire voler en éclats les vérités toutes faites, multiplier le sens de la vérité, élargir notre champ de vision sans pour cela nous tirer d'affaire.

La poésie et l'œuvre de Fondane sont inspirées d'une pensée inquiète que l'humour ne tranquillise pas.

Compte tenu de l'immensité de la pensée juive - qui n'est d'ailleurs pas la seule source de Fondane - on se limitera, dans cet article, à quelques rapprochements que permettent les textes de Benjamin Fondane, Léon Chestov et Vladimir Jankélévitch, pour montrer comment l'ironie provoque les ressources de l'impossible et donne au tragique une puissance paradoxale. Nous faisons l'hypothèse d'une pensée rieuse face à la tragédie, d'une ironie féconde dans son rapport à la poésie et au questionnement.

Il ne nous appartient pas ici de consolider l'image de ce que certains se plaisent à nommer l'" humour juif ", mais plutôt de

1 Benjamin Fondane, "Signification de Dada" dans Le voyageur n'a pas fini de voyager, textes et documents réunis et présentés par Patrice Beray et Michel Carassou, Paris/Toulouse, Paris-Méditerranée/L'Éther Vague, 1996, p. 36 [Dorénavant, $V$ ]. 
reconnaître l'ouverture qui est en son principe et qui résiste justement à toute catégorie en nous donnant le goût de l'absurde.

Par exemple, la contradiction chez Benjamin Fondane incite au questionnement, à une "exploration des antipodes" ${ }^{2}$; elle devient un mode de pensée qui fait éclater les cadres et nous engage comme son poète dans une lutte contre les évidences. Ainsi nous initie-t-il au mouvement de la pensée ou plus franchement encore, laisse-t-il transparaitre - selon ses propres termes - ce "trouble auquel nous reconnaissons la vie" ( $M F$, p. 11). L'exploration ne peut rester formelle; elle engage le poète à "revenir aux sources d'un monde qui n'a pas livré son mystère, et dont il est témoin parmi les hommes" ( $V$, p. 125). Fondane pressent que la vérité est toujours différente de ce que l'on croit. La poésie en témoigne. En 1929, il écrit:

La poésie n'est pas une fonction sociale mais une force obscure qui précède l'homme et qui le suit [...]. L'homme est un animal que la poésie pétrit dans l'argile, ou qu'elle fait sauter à coup de dynamite $(M F$, p. 23).

\section{La pensée rieuse}

Comme dogme, une pensée peut-être exhibée en guise de réponse à certaines questions. Comme rire, la pensée peut être offerte comme questions à certaines réponses. ${ }^{3}$

Voici ce qu'est la lecture aux éclats d'après Ouaknin qui nous parle du rire comme d'une mise en doute qui va créer du temps, mobiliser une myriade de questions, engendrer une pluralité d'interprétations:

Ce qu'on appelle l'humour talmudique, ou encore l'humour juif si l'on veut, c'est la mise en doute des vérités toutes faites, et d'abord la mise en doute de soi-même ( $L E$, p. 354).

Autrement dit, dans les termes de Jankélévitch:

Il faut se réveiller non seulement du monde, mais de soi. [...] tout le monde rit de nous, et nous sommes les derniers à le

2 Le mal des fantômes précédé de Paysages, traduit du roumain par Odile Serre et présenté par Patrice Beray, Paris/Toulouse, Paris-Méditerranée/L'Éther Vague, 1996, p.161 [Dorénavant $M F$ ].

3 Marc-Alain Ouaknin, Lire aux éclats. Éloge de la caresse, Paris, Seuil, Quai Voltaire, 1992, p. 52 [Dorénavant $L E$ ]. 
60

savoir; les hommes et les bêtes font déjà cercle autour de nous pour rire de notre perruque, de notre plastron et de notre grande conscience; mais nous croyons la nature plus drôle que nous-mêmes, et nous continuons à faire des jeux de mots! ${ }^{4}$

Cette grimace à la pensée n'en supprime pas le sérieux. Mais on ne peut après cela se prendre tout à fait au sérieux. Et après tout, en imposer ou même s'en faire imposer devrait faire sourire en nous la partie "soumise". Ainsi, l'humour assure-t-il un moyen de défense contre l'autorité, permet de la faire voler en éclats pour élucider notre rapport à l'autre, sans violence mais non sans audace:

L'humour offre à l'homme un moyen de déjouer la violence que d'autres voudraient lui imposer, de ridiculiser ceux qui voudraient lui oter son visage. Mais le rire, cette "lecture aux éclats" du Midrach et du Talmud, ne prétend pas opposer une vérité à une autre $(L E$, p. 354).

Alors l'ìronie dont la poésie de Fondane est chargée, devient un passage possible pour des "éclats" de Véritê: "À présent que le mystère a disparu de nos vies - c'est à la poésie que nous confions notre besoin d'absurde" (Carnet de route", $V$, p. 174).

"Sous le "lire" peut s'entendre le "rire": la pensée itinérante du Talmud est aussi un éclat de rire", suggère Marc-Alain Ouaknin ( $L E$, p. 354). Sans soulever les enjeux d'une tradition herméneutique millénaire, je signalerai brièvement un trait spirituel dans la pensée juive qui alimente une conception paradoxale du sujet:

Dans les textes d'inspiration cabalistique, il est souligné avec force que les trois lettres qui écrivent le mot Ani (Aleph-nounyod) écrivent aussi le mot Ayin (Aleph-yod-noun) qui signifie le "néant ", le "rien". 5

Privilégier le chemin, ou même l'effacement plutôt que l'image, c'est se préserver d'un enfermement. Ce qui ne justifie pas pour autant le fantasme de l'errance comme l'explique très bien Sibony:

C'est le fantasme de ceux qui vous chassent en vous accusant de n'avoir pas de lieu alors que vous symbolisez pour eux la fragilité de leur propre lieu [...] Errer, c'est ne pas trouver son

4 Vladimir Jankélévitch, L'ironie, Flammarion, 1964, p. 26.

5 D'après A. Safran, La cabbale, Payot, 1979, p. 288 et Samuel Trigano, Le récit de la disparue, Paris, Gallimard, 1977. 
chemin tout en le croyant déjà tracé, alors que c'est la recherche dont il est l'objet qui peut lui donner lieu ${ }^{6}$.

Ainsi le risque métaphorisé par le rire peut-il s'inscrire dans la pensée comme une "conscience en voyage"

L'homme [quil s'arrache à l'image de lui-même [...] refuse de s'enfermer dans le maintenant de la représentation, qui est abolition du temps ( $L E$, p. 363).

Ainsi le "vagabond humour" ne reprend son sens et son chemin que par cette mise en doute qui n'est peut-être pas sans allusion à l'interdit sur la représentation dans le judaîsme; ou plutôt qui fait de cet interdit la prescription d'un voyage sans fin. ${ }^{8}$

\section{Le rire vagabond}

Il y a aujourd'hui un lyrisme de la panique et si l'on peut souhaiter que la beauté soit convulsive, c'est qu'on désire la surprendre pendant qu'elle se forme et non pas lorsqu'elle est déjà achevée, morte ("Réflexions sur le spectacle", $L E$, p. 47).

La modernité a changé notre rapport au temps et au mouvement. On peut se demander si cette mobilité a réellement ouvert des voies dans la pensée et dans la sensibilité. Et quelles sont les conditions pour vivre et penser le passage, le vertige, le risque, maintenant que nous sommes comme des éclats de vie voyageant dans l'absence d'unité:

Émigrants, diamants de la terre, sel sauvage,

je suis de votre race,

j'emporte comme vous ma vie dans ma valise,

je mange comme vous le pain de mon angoisse,

[...]

- je ne saurai jamais me résigner. (MF, p. 113)

L'émigrant est à vif dans le risque qu'il prend du passage et son identité ne peut se façonner que dans l'effort qui le conduit et l'éconduit toujours vers la nouveauté, vers "un Triomphe dont s'est perdu le sens ", ainsi que le suggère Fondane:

6 D. Sibony, La jouissance du dire, Paris, Grasset, 1985, p.213.

7 Vladimir Jankélévitch, Quelque part dans l'inacbevé, Paris, Gallimard, 1978, p. 150 et suiv.

8 Ibid., p. 153 et suiv. 
62

- À quoi servirait notre vie,

à quoi nos batailles perdues, sinon à un Triomphe dont s'est perdu le sens, et pour porter en fraude aux hommes sous l'œil absent des douaniers une nouvelle beauté panique. ( $M F$, p. 108)

Il y a un rapport entre ce mouvement incertain (des émigrants) et cette "nouvelle beauté panique"; quelque chose de l'ordre de l'éclat que le mouvement enregistre; quelque chose qu'on ne peut ni arrêter ni enfermer sans l'anéantir. L'injonction d'un tel mouvement pour un "Triomphe" seulement hypothétique, révèle le caractère paradoxal d'une humanité potentiellement libre et cependant toujours servile. Le mouvement ne fait donc que nous préserver de la mort ou de la fixité qui n'est autre qu'une mort ignorée.

L'homme éthique, qui a refusé de s'enfermer dans un sens unique du monde, est toujours en route; il n'est jamais arrivé, il va ailleurs, toujours au-delà. ( $L E$, p. 354)

Dans Titanic, le XIII ${ }^{e}$ poème du "Poète et son ombre" indique négativement deux sortes de mouvement:

Nous n'irons pas plus loin que nous-mêmes! et :

Le voyageur n'a pas fini de voyager. (*Titanic " $M F$, p. 254)

Quel est le rire alors "dont s'est perdu le sens"? Plus que de l'homme, le rire est le propre de Dieu! Le poète a-t-il un autre choix que de Le servir et, tout au long de sa course, de recueillir des éclats de son rire?

La voix si singulière de Fondane insiste pour s'inscrire dans le temps et dans l'espace; comme si le chant voulait forcer l'histoire à retourner ses cartes; comme si la poésie pouvait se mettre à douter des versions établies de l'Histoire et réclamer son dû. Ainsi Fondane revient-il à Platon dans Faux traité d'esthétique, pour discuter ce rapport du poète à la vérité :

Que les dieux dussent "ravir l'intelligence" aux poètes, afin de pouvoir nous parler à travers eux, cela, certes, gênait la "probité intellectuelle. de Platon. Mais davantage était-elle gênée par l'intuition obscure, mais certaine, que les dieux ne donnaient l'intelligence qu'à ceux qu'il voulait perdre. Et c'est ainsi que, plus tard, Platon fut amené à déclarer que la poésie était 
éloignée de trois degrés de la vérité et de la sagesse, qu'elle remuait la partie mauvaise de l'âme - oubliant qu'il avait dit lui-même que le poète est irresponsable et que c'est le dieu qui parle à travers lui. ${ }^{9}$

La poésie de Benjamin Fondane, renouant avec les genres épique et dramatique, cherche à replacer l'inconnu dans le vécu, avec toute l'impatience que cela suppose:

[...] mais que font donc les vivants?

qu'attendent-ils pour mettre en marche les poulies? ("Ulysse ", $M F$, p. 91)

Cette exclamation ouvre un passage à l'intensité tout en révélant la frayeur du poète devant l'inconnu. Lorsque Fondane s'écrie: "Rire au nez de la tragédie ou disparaître", nous présente-t-il une alternative? Trace-t-il un chemin pour des questions sans réponses?

Les "lieux" de l'humour sont souvent des lieux de passage, ou des lieux en mouvement, ou des moyens de locomotion ( $L E$, p. 187).

Il existe une ironie inquiète de l'inconnu et franchement engagée dans le risque de soi, le risque d'une alliance avec l'impossible:

Mais pour que nous soyons capables d'entrevoir ne fût-ce que vaguement la possibilité de cette nouvelle dimension de la pensée, il faut que nous ayons le courage de chasser nos terreurs habituelles et que nous cessions de prêter l'oreille aux a priori de tout genre que nous souffle la raison. Et alors, "il n'y aura rien d'impossible pour nous . $^{10}$

Ce risque, une fois mobilisé contre l'ironie du pouvoir, n'a pas les moyens de rebrousser chemin. Il s'agit alors de "convaincre l'émigrant dans l'homme" ( $V$, p. 71$)$. Ainsi Fondane pouvait voir Rimbaud, indépendamment des satisfactions de la pensée spéculative qui voulait faire de lui un Voyant. Il écrit dans sa lettre à Victoria Ocampo, en s'excusant presque:

Je vous avais promis un saint, j'aurais honte de vouloir tenir parole; ne m'en voulez pas; je n'ai découvert qu'un voyou: mais de combien plus lumineux qu'un saint. $(V$, p. 59)

9 Faux traité d'estbétique, Paris, Plasma, 1980 (première édition 1938), p. 86.

10 Léon Chestov, Atbènes et Jérusalem, traduit par Boris de Schlozer et précédé de "I'obstination de Chestov" par Yves Bonnefoy, Paris, Aubier, 1993 (première édition 1967), "Bibliothèque philosophique", p. 317. 
64

Cette lumière du chant émané du bourbier renvoie au mystère de la poésie et à sa liberté plutôt qu'à sa beauté servile. Jouer à suivre ainsi du doigt toutes les contradictions, n'est-ce pas aussi contester aux apparences leur plein pouvoir?

Ce rôle contestataire s'inscrit dans le temps sans le nier, en lui donnant une autre allure... C'est en ce sens que l'ironie devance le souci, le met en cause; en ce sens aussi qu'elle casse le noyau insécable de l'instant, le fait germer...

L'ironie proteste contre le rationalisme statique et rend hommage à la temporalité de la vie; l'ironie dit à sa manière que toute l'essence de l'être est de devenir. ${ }^{11}$

N'oublions pas que le premier rire biblique fut celui d'Abram désormais Abraham, lorsqu'il apprit à l'âge de 100 ans, qu'il aurait - de sa femme Sarah qui en avait 90 ! - un fils : Yitshak, en hébreu le rire ${ }^{12}$.

\section{L'ironie dans son rapport à la vérité}

Nous sommes, il est vrai, pourris de pédantisme, de bavardage et de littérature, mais l'élan se porte ailleurs, et l'homme reste capable, grâce à l'humour, de sentir les choses avec rondeur et véhémence. ${ }^{13}$

Pas plus que Fondane ne s'est autorisé à considérer Rimbaud comme un voyant, Baudelaire comme un génie, nous ne pourrions le lire comme un prophète sans que son double s'anime d'une ironie parfaite et ridiculise un tant soit peu notre entreprise. Voilà pourtant que nos ambitions d'analyse sont démasquées! Nous sommes peut-être condamnés à vouloir du solide:

Je sais bien que personne ne cherche le poète avec le risque de le faire voler en éclats, ni Dieu avec le danger de le perdre.

("Mots sauvages", $M F$, p. 22)

La pensée juive, par l'image d'une vérité catastrophique dont les Tables volent désormais en éclats prévient peut-être par ricochet de cette ironie dont toute chose est obscurément accompagnée:

11 Vladimir Jankélévitch, L'ironie, op. cit., p. 196.

12 Ouaknin résume l'exégèse de Rachi à ce propos dans Lire aux éclats, p. 5354.

13 Vladimir Jankélévitch, L'ironie, op. cit., p. 196. 
Lorsque le je devient Anakbi, autrement dit se réalise de manière définitive dans l'écriture, il doit se proposer à la "brisure "faire éclater le Moi qui s'est identifié, qui a accepté le destin d'une identité ( $L E$, p. 360 ).

Le talmudiste est toujours à la recherche d'une désignification positive qui signifie que toutes les fois qu'il veut se mirer en luimême, le miroir se brise ${ }^{14}$.

La sagesse socratique "aboutit au savoir de sa propre ignorance" tandis que "l'ironie romantique n'exténue le monde que pour se prendre elle-même plus au sérieux" ${ }^{15}$.

Héritier critique de cette mosaïque culturelle, Fondane ridiculise le narcissisme de son voyageur qui devra accepter les épreuves mais pas les médailles:

Lorsque le voyageur, échappé au naufrage

parvint enfin dans l'île, ayant sauvé des flots

sa brosse à dents, sa pipe, son mal de foi et son

ancienne incrédulité au miracle,

le temps fondit comme un paquet de neige [...]

Le poète décrit le même voyageur pris d'une peur hallucinante, après une suite de malaises:

[Il eut] si peur, qu'il se jeta à genoux, qu'il pria

son dieu [...]

et se laissa tomber d'un rocher à la mer

juste un instant avant

qu'on ne lui eût donné l'esprit de prophétie. ("Au temps du

poème et poèmes épars ", $M F$, p. 374)

La prophétie, la voyance, le génie ne sont, pour Fondane, que les effets secondaires d'une autre expérience, à laquelle le voyageur - même ironisant - est tragiquement soumis.

Pour Fondane, Rimbaud, en renonçant à être le voyant, "piétine toute trace de miracle" $(V$, p. 35), n'assume aucun salut.

Cela doit nous enlever l'idée que la prophétie poétique ou le sacrifice historique justifient la beauté comme arme contre le désespoir. L'ironie chez Fondane signale bien que cela n'est qu'arrangement, foutaise et ne nous empêche d'ailleurs aucune-

14 Henri Meschonnic, Jona et le signifinant errant, Gallimard, 1981, p. 122, cité par Ouaknin, Lire aux éclats, p. 360.

15 Vladimir Jankélévitch, L'ironie, op. cit., p. 17. 
66

ment de rêver librement à la beauté et à l'unité des étincelles cabalistiques! Le rêve génère une liberté qui ne le lie plus à un quelconque sens de la souffrance ni de la magie; il est vertige sans promesse ni préavis:

L'ironie force l'injuste à être bien ce qu'il est, franchement, brutalement, pour qu'il en crève; elle le contraint à s'avouer luimême, car elle sait que ce sera sa perte. ${ }^{16}$

La conscience de soi devant l'image qu'elle se fait d'ellemême "respire plus légèrement quand elle s'est reconnue, dansante et grimaçante, dans le miroir de la réflexion", ce qui signifie que "l'humour n'est pas sans l'amour, ni l'ironie sans la joie, et ensuite que la lucidité ne se refusera pas à la ferveur " 17 .

\section{Désespérer le désespoir}

L'ironie du sort, apparemment, n'a pas la structure ambiguë du langage, et dans ce cas le ridicule tue:

Les dieux ont ordonné la mort

de ces bommes afin d'être sujets

de chants pour les générations à venir.

Homère

ET VOILA! - rétorque Fondane en exergue à son propre recueil L'exode ( $M F$, p. 259-324). Fondane ignorait en 1934 la résonance prophétique de son poème, qui donnerait une force presque compensatoire aux camps avec cette idée du sacrifice sur lequel repose l'art. Cela aurait pu nous satisfaire, si Fondane n'avait écrit par ailleurs la même année:

C'est clans le chant qu'on fabrique les meilleurs canons. Le fil de fer barbelé, c'est toute une chanson, et le gaz sifflote [...] L'escalier du temps ruisselle déjà de cadavres. Moi, vous, tout le monde. ${ }^{18}$

Comment Fondane a-t-il choisi de remettre de l'homme, autrement dit de l'affectif dans tout cela? Comment a-t-il préféré suivre sa soeur sur le chemin tout tracé de la mort.

16 Vladimir Jankélévitch, Lironie, op. cit., p. 10. Jankélévitch traite aussi du rapport de l'ironie à l'erreur et à la vérité à la p. 110 .

17 Ibid., p. 199.

18 Benjamin Fonclane, "Lever de rideau ", Le cabier bleu, 22 décembre 1933; reproduit dans le Bulletin de la société d'études sur Benjamin Fondane, $\mathrm{n}^{\circ} 5$. 
"L'arbre de la Science n'est pas celui de la vien. Cette contradiction, déjà soulevée par Chestov, était-elle au point de disparaittre?

Le degré second de l'ironie est possible à cause de cette distance prise par la conscience, distance qui, dans le temps et dans l'espace, s'affirme tout en se disséminant.

Par le langage nous pouvons encore devancer le cri. L'ironie, comme la poésie qui nous intéresse, condense en le désagrégeant l'effort de vérité. Cette structure ambiguë ne doit pas compromettre son but, dont un de ces plus grands luxes, une de ces plus grandes peines, qui est de transformer l'impossible en possible! Par la foi d'un sourire, d'une mélodie, d'un cri, que dissimule le chant...

L'ironie devance toujours le désespoir, nous dit Jankélévitch:

Elle fait la pirouette et, en moins de temps quiil n'en faut pour le dire, elle a déjà escamoté la cause de notre tourment; à la barbe du clestin nous voilà devenus jardinier, géomètre ou violoniste, et notre personne file en contrebande sous les masques les plus variés. ${ }^{19}$

"Talent propre à l'intelligence", "art d'effleurer" ${ }^{20}$; c'est à se demander si l'ironie entrevoit une quelconque profondeur aux choses? Seulement par un décentrement :

L'ironie, c'est de savoir que les îles ne sont pas des continents, ni les lacs des océans. [...] Grâce à l'ironie, les périples se bouclent et les problèmes se laissent circonscrire. ${ }^{21}$

Cette connaissance relative ne nous tire pas d'affaire puisqu'on ne peut douter du chant que par le chant, comme l'explique Fondane après quatre ans de silence:

Le jour où elle est revenue [...] comme un puits artésien, m'éclaboussant les muscles d'un arc-en-ciel, je compris de quelle misère profonde je sortais [...]. J'ai compris que la poésie, on ne pouvait s'en débarrasser, ni l'attraper au lasso quand on voulait. ("Mots sauvages", $M F$, p. 22)

L'ironie qui ne craint plus les surprises joue avec le danger [...] même elle se risquera à travers les barreaux [...] pour obtenir

19 Vladimir Jankélévitch, L'ironie, op. cit., p. 35.

20 Ibid.

21 Ibid., p. 27. 
68

l'illusion complète de la vérité; elle joue de sa fausse peur, et elle ne se lasse pas de vaincre ce danger délicieux qui meurt à tout instant. 22

"Les mots ont fait leur temps", jette Fondane ("Lever de rideau ", Bulletin...), ce qui témoigne d'une ironie sans désinvolture. Cet énoncé paradoxal mine la validité des définitions toutes faites. Il signale la frontière d'une ère déterminée d'un côté par l'impuissance des mots et, de l'autre, par leur résonance suprême "faute de mieux", à plus forte raison dans ce cas précis où leur perte est, pour ainsi dire, ironisée. On ne s'en débarrasse donc pas, mais au contraire, tragédie du sort, eux se débarrassent de nous:

J'ai mangé du fruit défendu et j'ai immédiatement su que j'étais nu, que le beau n'était pas moins douteux que la vérité... Les mots se sont d'un coup débarrassés de moi. Dans la nuit j'ai commencé à crier sans mots ("Paysage ", $M F$, p. 21).

Malgré la contradiction qu'impose à Fondane cette ironie du sort, la poésie s'affirme, et cela moins pour donner libre cours à de "secrètes aspirations" que pour "éprouver la résistance du monde" 23 :

Le monde est là peut-être, mais suis-je bien en lui?

Je passe et il ne reste rien dans le miroir,

pas même un trou

et j'ai beau m'exercer sur les mots hors d'usage

comme on redresse au marteau des clous qui ont déjà

servi, tordus, et qu'on les enfonce à nouveau,

il n'est pas de chanson donnée à tout le monde,

je ne peux pas fermer les yeux,

je dois crier jusqu'à la fin du monde:

"Il ne faut pas dormir jusqu’à la fin du monde"

- Je ne suis qu'un témoin ${ }^{24}$

L'ironie, n'est-ce pas aussi, en effet, se laisser traverser, dans l'œuvre comme dans la vie, par un vertige ou "un fou rire tragique devant l'incompréhensible " 25 en abandonnant derrière soi toute sécurité épistémologique? Catherine Mavrikakis parle d'iro-

22 lbid., p. 9.

23 Voir à ce sujet la présentation de Patrice Beray, "Une histoire de poète", dans Le mal des fantômes, p. 9.

24 Benjamin Fondane, poème dédié à Léon Chestov dans le recueil Ulysse, Le mal des fantômes, p. 156.

25 Catherine Mavrikakis, "Pour en finir avec tout éloge de la souffrance ", communication présentée à la Fifth Conference of ISSEI, Utrecht 1996. 
nie "comme figure qui peut empêcher la maladie et tout particulièrement le sida de prendre un sens de vérité de l'histoire, de dépassement de la maladie dans l'art "26. Chez Fondane, on reconnaît une gravité omniprésente qui donne corps à l'aventure du langage, comme s'il avait fallu une parure pour notre nudité d'argile honteuse devant le gouffre.

Sur la pointe du désespoir métaphysique, l'homme a beau vouloir dormir ou faire de l'alpinisme, il est livré pieds et mains jointes à la souffrance éternelle. Cependant, il vit, et peut, par abondance de vie, vouloir ignorer sa souffrance. Qu'il l'accepte de bon coeur ou la défie, l'oeil méchant, ce sera la même chose. Quel est le moyen de s'en sortir? car il faut que l'homme s'en sorte. L'homme ne peut se passer de la souffrance mais il a besoin de la joie. ("Signification de Dada "V, p. 33)

Nous reconnaissons ici les paradoxes de la poésie selon Fondane, qui enchaîne en citant Freud:

C'est alors que commence la grande série de ces méthodes qui se sont formées dans l'âme humaine pour se soustraire à la souffrance, série qui commence par la névrose et la folie et dans laquelle sont comprises, l'ivresse, la contemplation excessive de soi-même, l'extase, l'humour.

"Parmi toutes ces méthodes, il faut choisir ou être choisi", bien que ces différentes possibilités puissent aussi entraîner les deux risques:

La terre n'avait plus d'odeur

Le sang me jaillissait du nez ${ }^{27}$

Voici deux vers qui surprennent comme un coup de poing dans le visage de celui qui se redresse. La métaphore qui suit en demeure boiteuse, ironique, voire sordide:

La volonté avait des cors aux pieds

La question est aussi de savoir si l'on peut faire un chant à partir de l'horreur:

26 Le fou rire de Foucault devant le sida et le commentaire ironique cle Guibert, toujours selon Mavrikakis, "deviendraient lentement au fil des pages un plongeon au coeur d'une insécurité tragique.

27 "Non-Lieu ", 1978, reproduit dans Cent poèmes pour la libertê, choisis par Ahmed Ben Othman et Jean-Pierre Damon, Amnesty Internationale, Le Cherche-Midi, collection "Espace", 1984. Une autre version du même poème existe dans "L'Exode. Super Flumina Babylonis", reproduit dans Le mal des fantômes, p. 286-287. 
figures sans visages qui haïssez

les feuilles, les voix et les chansons

Le poète nous a livré un chant que rien ne peut balayer de la terre, qui, même perdu "comme une feuille morten, ne se perd pas vraiment.

N'est-ce pas créer une faille dans la souffrance déjà-là que d'en faire un chant qui en porte le cri? Et ainsi de se donner du temps: temps pour que se développe la place du témoin, de l'interlocuteur, de l'autre et, avec elle, une "infinité de points de vue, en sorte qu'ils se corrigent mutuellement "28. Et surtout que notre humanité demeure par le chant, puisque c'est le seul choix que nous ayons:

Envoi

Que l'on nous brûle ou que l'on nous cloute

et que ce soit chance ou déveine,

que voulez-vous que ça nous foute?

Il n'est de chanson que l'humaine ( $M F$, p. 300).

Si l'alternative revient au même, on peut s'apercevoir ici que c'est le chant qui fait la différence, "la différence qui fait le livre" - dirait Duras ${ }^{29}$, que notre humanité n'est peut-être pas déterminée par ce chant, mais que l'inverse n'est pas impossible:

Nous avons tous peur, une peur panique, de cet univers qui grouille en nous et hors de nous; et c'est pourquoi nous crions que ce qu'il nous faut, avant tout ce sont des idéals. C'est pourquoi aussi nous aimons la poésie... ${ }^{30}$

Ainsi le poète peut se maudire lui-même de sa découverte, si bien qu'il n'a même plus besoin des autres pour qu'on le pointe du doigt :

La conscience, en se moquant de soi, ne détruirait-elle pas l'instrument même de la moquerie. On ne lui fait pas peur : c'est là sa force mais aussi sa faiblesse; elle est consciente à l'extrême avec ses vastes zones d'inconscience. ${ }^{31}$

J'ai grimpé le plus haut que j'ai pu et je n'ai pas trouvé la hauteur

28 Vladimir Jankélévitch, L'ironie, op. cit., p. 32.

29 Voir Les yeux bleus cheveux noirs, Paris, Minuit, 1986.

30 Benjamin Fondane, Baudelaire et l'expérience du gouffre, Bruxelles, Éditions Complexe, 1994, p. 18.

31. Vladimir Jankélêvitch, L'ironie, op. cit., p. 25. 
- Où est-elle donc l'altitude?

"Monte", nous a-t-on dit, "grimpe", nous a-t-on dit,

- et j’ai senti soudain que se glaçait la vie

elle quittait ses feuilles, ses fleurs et ses chansons

elle se retirait sous le vent

sous l'âpre vent des vérités hostiles -

mais où est-elle donc l'altitude?

"Il faut nous élever au-dessus de nous mêmes!"

Le poème signifie bien le vertige d'une mort anticipée: celle de Fondane, arrêté en mars 1944, puis transféré de Drancy à Auschwitz où il est gazé en octobre de la même année. La pluralité des voix tout au long du poème évoque la tragédie grecque. Ces voix font ici également songer aux injonctions rabbiniques qui disent sans cesse que l'être humain est infiniment perfectible:

Je n'aurais jamais cru que la barbe des sages

était si dure à traverser.

c'étaient des fils de fer barbelés, ils mordaient jusqu'au sang.

- "Plus haut, plus haut encore! 32

Gravité d'une sagesse tournée en ridicule. La seule synthèse possible résonne tragiquement dans l'allitération (barbe barbelés) et nous rend capables d'entendre sans la gloser cette ironie du sort qui conduisit les sages et les moins sages au crématoire.

SIN

Le cri que l'on pourrait crier

Il n'est pas ici, pas encore

Il rôde autour de quelque bouche

il sollicite une salive.

THAV

Sa figure m'est inconnue-

mais s'il mendiait dans la rue

mon frère, mon frère le Cri,

je sauterai bas de mon lit

et lui baiserai les pieds nus! ("L'exode ”, $M F$, p. 268)

Si l'erreur est lâcheté, comment donc ne pas crier, ne pas s'éveiller! L'inadéquation qui persiste entre la connaissance et l'Histoire nous met au défi de comprendre, met en demeure le langage et le chant; relégués au devant ou à la traîne, ils ne nous sauvent pas d'un asservissement sempiternel.

32. "Non-Lieu", op. cit. 
72

Témoin du désastre historique, Fondane fait de nous un témoin, par le cri d'alarme tant poétique que philosophique qu'il nous lance. Contre Platon, il nous fait repenser toute l'importance du chant:

Les dieux furent donc supprimés; les poètes furent asservis au Bien; la dialectique vainquit; elle osa même se faire passer pour le seul interprète * autorisé " des dieux.

Et pourtant le charme continua. Il rappelait aux philosophes l'existence d'une autre vérité, qui transcende l'acte intellectuel et la pureté morale, une vérité pour qui l'existence existe, et la chair, et les images, pour qui Dieu lui-même est image, vision, et non acte pur d'entendement vide... ${ }^{33}$

Cette autre vérité est pour Fondane celle de la chair et du sang; de l'affectivité. Réparatrice? édifiante? Certes pas; le chant dit l'importance d'un témoignage autrement resté inaudible. Le cri soutenu par le chant nous parvient alors, dans la gangue de sa force et hors de sa morbidité. Cependant, il ne présage en rien des temps meilleurs, bien qu'il en installe la possibilité au second plan, tandis que le premier plan reste envahi par la question de notre erreur dans une souffrance que seule, peut-être, la lâcheté nous impose. Notre fonctionnalisme serait-il un avatar de notre pensée?

Mais à y réfléchir deux fois, c'est peut-être calomnier l'homme, l'homo sapiens, que de lui attribuer une si exclusive et intolérante passion de l'utile. Il y a peut-être autre chose qui le démange de plus profond, de plus tenace, et dont la solution utilitaire n'est qu'un prétexte adroit. ${ }^{34}$

L'ironie du sort pourrait donc revenir à cette contrepartie; car le chant par le corps lui aussi en impose, au moins autant que le péché! Le chant donc nous amène donc à infirmer l'inexistence de l'impossible ${ }^{35}$.

Si la souffrance est évidente, le chant et la joie doivent être provoqués, comme l'esprit dans la lettre, comme l'ironie dans notre conscience, comme Dieu dans la chair. Ainsi pour Fondane, la question de Dieu n'est autre que la question de l'homme. En ce sens, l'ironie n'est pas cruelle. Ce cri corrosif chez Fondane

33 Benjamin Fondane, Faux traité destbétique, op. cit., p. 86.

34 Ibid.

35 Voir Léon Chestov, op. cit., p. 320 et suiv. 
provoque une ouverture pour l'impossible et n'est qu'une preuve de vérité poétique. Si la poésie n'est pas pour lui magie, elle est peut-être, comme l'ironie, mode de connaissance:

À l'encontre des lois [...] le poème n'apportait qu'un alibi. Le poème: quelque chose qui modifie la réalité? Non... Quelque chose qui me modifie... Moi? Mais qui? Et qui suis-je? (Mots sauvages ", $M F$, p. 22)

Le miroir vole encore en éclats...

\section{L'ironie fraternelle}

Bien que toute connaissance n'ironise pas ouvertement sur son objet, on peut appeler la connaissance une ironie naissante, un sourire de l'esprit. ${ }^{36}$

Et ce sourire s'adresse au témoin, au témoin du témoin... Il existe dans l'ironie une tristesse affectueuse et un peu narquoise, telle qu'on trouverait chez Baudelaire une "valse mélancolique" pour répondre à ce "coeur tendre, qui hait le néant vaste et noir! "; pour répondre à cette peur qui grouille en nous, cornme l'explique Fondane - et trouve à peine appui dans cette "Harmonie" du sonnet, si géniale soit-elle! Baudelaire nous permet au moins de devenir le spectateur souriant et attendri de notre bonne volonté et de lorgner du coin de l'œil cette fin de bal; cette part maudite de l'existence que nous nous attachons à trouver bonne et harmonieuse, comme nous l'eussions voulu d'un Dieu dont la perfection fut d'intention et non de fait.

L'art, le comique et l'ironie deviennent possibles là où se relâche l'urgence vitale. ${ }^{37}$

Chez Fondane, il existe une forme d'attachement second, authentifié par la foi que le poète porte au chant, quand bien même cette foi serait aussi objet de doute et de dérision. Cet attachement est une forme de respect face à un mystère qui nous provoque, qu'il s'autorise à provoquer, tout comme son maitre:

Chestov démontre l'impossibilité de la connaissance par ses propres moyens (la garantie qu'aucun Dieu ne nous trompe)...

("Carnets de travail 1940-1944 [extraits] $V$, p. 173-174)

36 Vladimir Jankélévitch, L'ironie, op. cit., p. 22.

37 Ibid., p. 9. 
74

L'ironie est peut-être la preuve que les carcans sont à la mesure des risques que nous voulons ou refusons de prendre [seuls].

La causticité de Fondane nous met en situation, non pas seulement de lire, d'interpréter, mais aussi de prendre position, et au besoin de renoncer à une certaine sécurité épistémologique et morale.

L'ironie, anonyme chez Socrate, éprouve le besoin de se montrer hirsute, crasseuse et agressive; au lieu d'analyser les idées, elle préfère les aphorismes. ${ }^{38}$

On peut soupçonner alors que la poésie excède le champ de l'expérience langagière et conceptuelle, qu'elle existe aussi pour nous faire prendre un risque, risque de soi, risque de l'inconnu, de l'incompréhensible.

Et si par hasard l'ironie était initiation à la générosité? [...] l'ironiste s'arrange (suprême habileté!) pour que son partenaire assure lui-même la compensation; l'ironiste fait faire par l'autre tout ce l'autre peut faire de lui-même [...] ainsi la litote ironique ne sert qu'un seul maître, - le Vrai; c'est la vérité impersonnelle par delà du Moi et du Toi qui commande l'amoindrissement désintéressé; l'amour extatique. ${ }^{39}$

L'ironie comme heuristique permet de maintenir le clair-obscur du paradoxe tout en "[choisissant] la justice" ${ }^{40}$. Elle privilégie le mouvement et l'ouverture dans la pensée, en intégrant ce risque dans le temps, comme un voyage de la pensée s'opposant à l'Histoire, à ses relations de causes à effets et à son principe de non-contradiction. L'ironie existe dans cette subtilité du temps qu'elle dérobe à l'Histoire. Contre les apparences, tout contre, la poésie dialogue avec le Vrai. Car, ce que nous dit l'ironie, chez Fondane, c'est que le langage, contrairement aux événements, n'est pas irréversible; qu'à cet endroit, au moins, nous avions le choix et le pouvoir - non pas de contraindre le monde à n'être que ce qu'il est - mais de lui rendre sa liberté de devenir.

Entre "son frère, le Cri", et la fumée qui l'a fait disparaître, il y a un Fondane qui se moque de bien des choses, mais qui cherche partout encore des frères et soeurs, convaincu que plus que d'un public, "la poésie a besoin d'amis".

38 Vladimir Jankélévitch, L'ironie, op. cit., p. 16.

39 Ibid., p. 90-91.

40 Ibid., p. 39. 
Car [nous pouvons le conclure avec Jankélévitch] le but de l'ironie n'était pas de nous laisser macérer dans le vinaigre des sarcasmes ni, ayant massacré tous les fantoches, d'en dresser un autre à la place, mais de restaurer ce en quoi l'ironie ne serait même pas ironique : un esprit innocent et un coeur inspiré. ${ }^{41}$ 